\title{
Cultura de la resistencia y estética del deterioro. Marta Traba y la articulación conceptual de la crítica artística latinoamericana
}

\section{Culture of resistance and aesthetics of deterioration. Marta Traba and the conceptual articulation of Latin American art criticism}

\author{
Matías Marambio de la Fuente ${ }^{1}$ \\ Universidad de Chile. Santiago, Chile. \\ matias.marambiodlf@gmail.com.
}

\section{Resumen}

El siguiente artículo tiene por objetivo examinar el pensamiento de la crítica de arte argentinocolombiana Marta Traba mediante una discusión de dos conceptos: la cultura de la resistencia y la estética del deterioro. Se realiza una reconstrucción del entramado teórico y contextual en el que emergen ambas categorías, entendiéndolas como parte de un desarrollo crítico de considerable complejidad. Sostengo que Traba realiza un aporte original en su tratamiento de tradiciones teóricas euro-norteamericanas y latinoamericanas, lo que le permitió desarrollar una mirada crítica capaz de poner en perspectiva socio-histórica la modernización de las artes visuales en América Latina desde la postguerra hasta principios de los setenta.

Palabras clave: Marta Traba, crítica artística, América Latina, historia intelectual.

\section{Abstract}

The following article wishes to examine the thought of Argentine-Colombian art critic Marta Traba through a discussion of two concepts: the culture of resistance and the aesthetics of deterioration. The paper makes a reconstruction of the theoretical and contextual network in which both categories emerge, understanding them as part of a critical development of considerable complexity. I contend that Traba makes an original contribution through her elaboration of theoretical traditions stemming from Europe, North America and Latin America, which allowed her to develop an approach capable of setting the modernisation of Latin American visual arts from the post-war period until the 70 s into socio-historical perspective.

Keywords: Marta Traba, Art Criticism, Latin America, Intellectual History.

1 Becario CONICYT. El presente artículo está basado en mi tesis de Magíster en Estudios Latinoamericanos, "Campo intelectual y artes visuales: Marta Traba y la formación de una crítica artística latinoamericana". 
La historia del pensamiento latinoamericano parece constituir un mapa cuyos hitos y fronteras nunca acaban de dibujarse. Cada intento por construir genealogías, redes de influencias, series de temas o instancias de intercambio intelectual se confronta, a poco andar, con la necesidad de poner en cuestión lo que queda dentro y lo que se descarta. Dentro de dicho escenario, es importante reconocer que la historia intelectual del continente ha tendido a reconocer primariamente las prácticas que refieren a la escritura (cf. Rama, La ciudad letrada) por sobre otros registros. El objetivo de este artículo es indagar en la figura de Marta Traba (1923-1983), una de las intelectuales más relevantes del mundo de las artes visuales latinoamericanas del siglo $\mathrm{XX}^{2}$. Para ello realizaré una discusión de dos categorías fundamentales en su obra (cultura de la resistencia y estética del deterioro), con miras a desentrañar el tinglado conceptual y socio-histórico que en ellas se anuda y especificar las contribuciones de Traba a la creación de un marco de pensamiento para las artes visuales latinoamericanas de su tiempo. Desarrollaré mi argumento de la siguiente forma: primero realizo un breve excurso sobre la forma en que entiendo el estudio de los conceptos como estrategia investigativa en la historia intelectual (I); posteriormente procedo a la reconstrucción de los conceptos de cultura de la resistencia (II) y estética del deterioro (III) merced a un análisis de los textos de Traba en el que se identifican sus referentes intelectuales y se propone una lectura del armazón que se desenvuelve en dicha obra ${ }^{3}$.

Este artículo toma como su materia fundamental el estudio de los conceptos como estrategia metodológica para reflexionar sobre la historia de la crítica artística latinoamericana. Corresponde, en un gesto auto-reflexivo, hacer una breve aclaración teórica respecto de cómo se piensan los conceptos. Se trata, ante todo, de una aproximación más bien sinóptica -en el sentido que le da Martin Jay ("Fieldwork and Theorizing"; “Two Cheers”) -, que intenta combinar sincronía y diacronía; esto es, la caracterización de la entidad categorial a la vez que su trayectoria. Al mismo tiempo, comprendo a los conceptos en su dimensión cultural, más que en el sentido filosófico. De esta forma, los conceptos son una manifestación de intersubjetividad, "distorsio-

2 La visibilidad de su trabajo tras su muerte (un accidente aéreo que le costara la vida a ella, Ángel Rama, Jorge Ibargüengoitia y Manuel Scorza) no se condice con aquella que tuvo en vida, lo que ha sido explicado en parte por los proyectos disímiles que se afincan en la crítica artística latinoamericana a partir de los ochenta. Ver Ramírez (36-40); Jara $(29,90)$.

3 El trabajo de identificación de los referentes de Traba no es, con mucho, mi contribución original. De los textos que examinan su pensamiento (ver Giraldo; Jara; Berríos; Pizarro; Ramírez) creo que el aporte más decisivo es el realizado por Florencia Bazzano-Nelson ("Cambios de margen”; “Theory in context"), quien es responsable de la anotación de Dos décadas vulnerables en las artes plásticas latinoamericanas, 1950-1970 en su segunda edición. Cabe mencionar, también, la investigación más reciente de Fabiana Serviddio, quien ha explorado la obra de Traba en el marco más amplio del campo artístico de los setenta (193-241). 
nan, desestabilizan y sirven para dar una inflexión al objeto" (Bal 35). Como tales, son, ellos mismos, artefactos culturales de factura teórico-lingüística; se manifiestan concretamente (en especial en el ejercicio de la crítica y en los ámbitos intelectuales) como palabras, pero son algo distinto de las palabras. Al decir de Reinhart Koselleck, esto se relaciona con el problema de la uni- y la polivocidad de las palabras: "Una palabra puede, pues, -en el uso- volverse unívoca. Un concepto, por el contrario, debe permanecer polívoco para poder ser concepto" (119) ${ }^{4}$. Por ende, "un concepto agrupa la multiplicidad de la experiencia histórica y una suma de nexos teóricos y prácticos en un contexto que, como tal, sólo está dado y se vuelve realmente experimentable por medio del concepto" (Koselleck 120). Concepto y contexto -o "estado de cosas", siguiendo a Koselleck (121)- entablan una dialéctica en la cual el primero aprehende y da cuenta del segundo, sin poder abarcarlo del todo. A su vez, aquella realidad no puede existir sin más, carente de conceptos; se volvería una realidad sin significado alguno, un mundo en el cual sería imposible entablar cualquier tipo de comunicación. Si los pensamos dentro del ámbito que nos compete -la crítica artística-, los conceptos funcionan no sólo como condensación de experiencia histórica, sino como teorías en miniatura que median entre la crítica y su objeto (Bal 37). Se trata de entidades culturales que, al trabajar sobre otras entidades culturales, permitiendo que los intelectuales las describan, analicen y relacionen, mutan ellas mismas, a la vez que fuerzan la transformación de otros conceptos.

En el caso de Traba, cabría señalar que las hebras conceptuales mayores de su pensamiento anudan desarrollos teóricos de, al menos, tres tradiciones identificables: a) la crítica cultural latinoamericana desarrollada bajo la forma emblemática del ensayo, del siglo XIX hasta su tiempo; b) los aportes de las ciencias sociales latinoamericanas de la segunda mitad del siglo XX; c) las teorizaciones realizadas en Europa occidental y las zonas desarrolladas de Norteamérica, en especial aquellas aproximaciones sociológicas a los procesos culturales, sea bajo un signo crítico -como Herbert Marcuse, Theodor W. Adorno o Henri Lefebvre- o bien con un espíritu más complaciente -como Marshall McLuhan-. He elegido sólo dos conceptos clave, debido a su mayor peso y presencia dentro de la reflexión de Traba, en especial en Dos décadas vulnerables, que vendría a representar uno de los más importantes hitos dentro de su obra (cf. Bazzano-Nelson "Cambios de margen"). Otras categorías, que se desarrollan tanto en Dos décadas vulnerables como en textos anteriores y posteriores, pueden vincularse a estas dos, sin perjuicio de que ellas reclamen asociaciones que les son particulares. Sostengo que la contribución de Traba a la historia intelectual latinoamericana pasa por el trabajo de urdimbre, por un anudamiento que se mueve en dos esferas: 1) la asociación de conceptos entre sí, con sus tradiciones, cargas semánticas y teóricas diferenciadas; y 2) el ensamblaje de conceptos - propios y "ajenos"- y obras. El alcance

$4 \quad$ Las traducciones de textos del inglés y el alemán son mías. 
de este artículo se refiere sólo a la primera dimensión (vínculos entre conceptos), lo que no quiere decir, por su parte, que la elaboración teórica prescinda por completo de las obras; por el contrario, la apuesta de Traba es justamente la formación de una crítica que responda adecuadamente a los objetos que tiene por delante. Hablando de las figuras de Bacon, De Kooning, Cuevas y Dubuffet, reconoce la fuerza indiscutible de las imágenes, su capacidad de anular la teorización: "Dejé entonces de superponerles ideas, de convertir a los cuadros en malabares de mis pensamientos" (Los cuatro monstruos 11).

\section{II}

El entramado teórico construido por Traba en los sesenta constituye un punto de inflexión notorio en la historia de la crítica de arte latinoamericano. Para BazzanoNelson "el nuevo marco conceptual elaborado por Traba funcionó como un puente que la ayudó [...] a articular su visión crítica y provocadora por medio de lo que fue, en su momento, uno de los andamiajes teóricos más sofisticados puestos en circulación en la crítica de las artes visuales del hemisferio" (“Cambios de margen” 10). Se trató tanto de una "actualización” de repertorios interpretativos -en diálogo con los últimos desarrollos de la teoría social latinoamericana, la semiótica, la teoría de las comunicaciones y la crítica cultural- como de una comprensión de conjunto del proceso plástico continental que era capaz de hacerse cargo de la politización experimentada desde fines de los cincuenta en múltiples esferas de la vida latinoamericana ${ }^{5}$. Cierto, Traba no estuvo sola en este esfuerzo, cosa que ella misma reconoció (ver "La tradición" 21-36) ${ }^{6}$, y sus coetáneos tienen a su haber logros que sería necesario explorar tanto individualmente como en un enfoque de conjunto.

Dentro del pensamiento de Traba la categoría de resistencia, también bajo la denominación cultura de la resistencia, abre la puerta a una comprensión de este doble articulado que acabo de describir (actualización teórica y comprensión histórica). Al situar una lectura de la historia de las prácticas culturales latinoamericanas desde el siglo XIX hasta principios de los setenta, Traba sostiene: "La obstinación de la cultura por perforar el problema de la dependencia parte, desde luego, de la confianza de vencerla y superarla, y de la certidumbre de que, dentro de ella, nunca se podrá aspirar a las formas modernas de libertad" ("La cultura" 37). Arremeter contra la dependencia, reclamar su extinción por vías que le sean propias a la cultura, remite a un terreno que desborda de obstáculos, pues plantea una pregunta de compleja resolución: ¿cómo

5 Sobre el proceso de politización del campo artístico latinoamericano ver Giunta; Schwarz; Ridenti; Longoni y Mestman (Del Di Tella).

6 Aquí Traba realiza una revisión de los aportes más recientes de la crítica artística, además de proveer una bibliografía a la fecha (1977). 
afirmar autónomamente una particularidad de/desde América Latina? La dificultad no sólo la intuye Traba, sino que la señala con toda agudeza: "conseguir mediante la autonomía y la liquidación de la dependencia, una identidad, significaba y significa para el trabajo artístico y literario un delicado problema de utilización de fuentes culturales y fuentes de lenguaje" ("La cultura” 38). En un examen que la lleva más allá del período definible e identificable como moderno, pondera las relaciones entre Europa (y, más tarde, Estados Unidos) y América Latina en términos de traspasos y readaptaciones de lenguajes plásticos: "el arte fue recibido como lengua y también como habla, maleable y manipulable. Tanto en el terreno de la creación culta, siempre más obsecuentes con el modelo, como en el de los espontáneos y primitivos, los trabajos latinoamericanos revirtieron sobre el campo emisor" (Dos décadas 15).

En este gesto de relectura histórica, Traba sitúa de nuevo a las individualidades que le sirven para sustentar su tesis de una tradición con vocación de afirmar la autonomía cultural de los contingentes humanos de América, lo que la emparenta con al menos dos de las figuras más notorias de la crítica literaria de su tiempo: Ángel Rama $^{7}$ y António Cândido, a la vez que con la semiótica de cuño estructuralista que hacía su real en este lado del Atlántico a fines de los sesenta y principios de los setenta. Sobre esto último, la distinción lengua/habla, ya introducida por Saussure, remite a la diferencia de estatuto lingüístico entre el lenguaje como abstracción y el lenguaje como concreción (entre la rigidez de la estructura en su "repliegue" sincrónico y la fluidez de la historia en su "despliegue" diacrónico). En su analogía, Traba enfatiza sobre todo este último aspecto, pues sería el momento mismo de transformación de un código foráneo en un repertorio de recursos usables por una comunidad que no ha originado ese código, pero cuyo uso reviste la posibilidad de expresar contenidos que les sean propios. Más aún: trabajaría la cultura latinoamericana de tal modo la lengua que sería capaz de transformar no sólo los léxicos, sino las reglas mismas que conforman un lenguaje artístico.

Ahora bien, el entramado conceptual en el que se inscribe la noción de cultura de la resistencia remite este proceso adaptativo a una trayectoria cuyo itinerario implica un conjunto de premisas que le son caras a Traba, y que indican ya la heterogénea y compleja composición de su instrumental analítico. Clave entre estos postulados es "considerar que la palabra escrita, el pensamiento emitido o la obra de arte expresada, constituyen una forma especial de poder dentro del grupo social al encarnar las aspiraciones de dicho grupo" ("La cultura" 38), con lo que se afirma que "el intelectual ve

7 Ver Ángel Rama, Rubén Darío. Que Rama viene a cambiar las consideraciones hasta ese momento imperantes sobre el modernismo es un punto que vale la pena reiterar. Eventualmente se podría esbozar la hipótesis de que tanto Dos décadas vulnerables como Rubén Darío significan puntos de inflexión para las respectivas "disciplinas" de Traba y Rama, pero eso excede mis capacidades actuales. De todos modos, resulta interesante notar la cercanía editorial -fácilmente previsible, por lo demás, debido al expatriamiento de ambos-en la publicación del estudio de Rama y la aparición del ensayo de Traba que anticipa su texto de 1973: Arte latinoamericano actual. Cf. BazzanoNelson (“Cambios de margen" 25-26). 
el proceso social de manera distinta al resto, no por superioridad o inferioridad, sino por simple división del trabajo" ("La cultura" 45). Reclamar una especificidad para la acción cultural remite, indirectamente, a las observaciones que ya había hecho Pierre Bourdieu sobre la autonomización del campo intelectual. De forma más o menos directa, Traba hace suyo el esquema de las relaciones dentro del campo cultural, en particular las referidas a la confrontación entre ortodoxia y heterodoxia como fuerza dinamizadora de la cultura, y aquilata, también, el peso que la tradición cultural tiene al estructurar un espacio para el arte.

Pierre Bourdieu llama "familias de cultura" o "familias de pensamiento" a la relación que los individuos que intervienen en la cultura de un país mantienen con legados de las generaciones precedentes, con significaciones "más o menos consagradas, más o menos nobles, más o menos originales, más o menos marginales", que representan un vasto cuerpo intelectual al cual el artista puede remitirse sin cesar, bien sea para aceptarlo o rechazarlo (Dos décadas 154).

En paralelo, se puede afirmar que la derivación de la particularidad de la acción cultural responde, a su turno, a una peculiar división del trabajo; premisa que tiene sus arraigos, entre otros, en la crítica cultural de Theodor Adorno ${ }^{8}$. Aquí, sin embargo, me parece importante señalar que otros elementos conforman el instrumental analítico de la argentina-colombiana, pues estas posturas sobre la división social del trabajo tienen un arraigo en su obra temprana. Su fundamentación del arte moderno como proyecto de individualidades figura al artista como "un ser eminentemente apolítico, antisocial, desinteresado de lo contingente, un ser que está en el medio de la historia como una isla inquietante" ("El genio anti-servil" 149-150).

Con esta leve digresión he querido subrayar cómo un concepto se articula a partir de un proceso casi nunca continuo, que elude el monolitismo que, las más de las veces, está implícito en la división del pensamiento de una autora en "obra temprana" y "obra madura". Consecuentemente, el delinear las coordenadas del concepto de cultura de la resistencia reclama tener en cuenta que estos elementos previos no existen como meros "antecedentes", sino que conforman hitos cruciales dentro del proceso de constitución de un concepto a lo largo del tiempo, actualizándose más tarde como estratos latentes. De este modo Traba puede explicar, ya en los setenta, la peligrosidad del artista a partir de su posición de privilegio socio-cultural, la cual produce tanto una sensibilidad contrapuesta a los intereses de las clases dirigentes como un desgarro en su constatación de no-pertenencia a las clases populares (Dos décadas 152-153). Si es que no hemos de caer, dice Traba, en la exigencia del socialismo ortodoxo, no podemos eludir esta separación entre público y artistas, al punto

8 Por ejemplo: "Valéry proclama la contradicción del trabajo artístico como tal con las condiciones sociales de la producción material hoy dominantes" (Adorno, Crítica cultural 195). 
en que el arte como creación cultural específica no circula por los amplios segmentos que el artista buscaría representar. La cultura de la resistencia, entonces, signaría sus esperanzas en una re-conexión entre artista y comunidad como apuesta de afirmación de una particularidad latinoamericana. Me permito citar aquí in extenso:
La única manera de que el público deposite su confianza en el artista y lo rodee y reconozca es consiguiendo que el artista revele los anhelos de esa comunidad, cualquiera que sea la desesperación, la peligrosidad o la vehemencia de dichos anhelos; cualquiera que sea la "herejía" que produzcan respecto a la "ortodoxia" de los centros emisores.
La única manera de que el artista lleve a cabo tal tarea es situándolo frente, dentro y por encima de dicha comunidad, de modo que la pueda observar, vivir y comprender, desenajenándose así de la obediencia o la obsecuencia que lo liga al centro emisor.
La única manera de que el arte tenga relativa autonomía es que se genere en esa relación; y que el artista, consciente de los vacíos por donde atraviesa y de la precariedad de las situaciones culturales, trate de levantar, desde esa relación, una estructura de sentido (Traba, Dos décadas 156-157).

Traba condensa aquí un proyecto que describe con mayor detalle e insistencia en otros pasajes de su obra, y lo que me parece justo es des-glosar algunos de los aspectos más relevantes en lo que respecta a la construcción de la categoría de cultura de la resistencia. Si en el primer párrafo se postulan las relaciones sociales que enmarcan la situación actual (el presente opacado por las desiguales relaciones centro/periferia) y la deseada (la representación de los deseos de un colectivo) del artista latinoamericano, hacia el final podemos notar cómo la utopía artística tiene por horizonte la ya mencionada superación del estatuto dependiente de la plástica continental. No habría un camino propio que renegase de las aspiraciones sociales mayoritarias o que buscara representarlas mediante el traslado de formas foráneas de conciencia y praxis artística; la re-inserción del artista en el cuerpo social debe hacerse cargo de esta dimensión estético-política, o no será. Sin embargo, me parece que el argumento más interesante se instala al final, pues, contra una defensa despolitizante y aséptica de la autonomía artística, Traba opta por enfatizar la autonomía del arte en términos de culturas que sostienen relaciones de poder asimétricas. En este gesto se vale de los aportes de la teoría social latinoamericana que problematizó las relaciones de dependencia en el marco de una relectura del esquema centro/periferia ya desarrollado por la CEPAL (cf. Roitman). Resistir es fundar una autonomía artística que rebasa la mera auto-referencialidad de los lenguajes artísticos -la legalidad interna de la obra-, proyectándose en un horizonte que se involucra de lleno en una totalidad social cuyas contradicciones se despliegan en el plano global, pero que implican -en lo concreto- al artista como sujeto frágil y expuesto a las trampas que tiende una cultura enajenada. El resultado de esta operación sería, a su vez, el construir una "estructura de sentido" 
que es tratada en otros momentos como el levantamiento de un "código general", tal y como lo piensa Lefebvre (102-104), tanto para pensar los efectos estéticos del arte metropolitano y sus consecuencias en Latinoamérica, como para elaborar parte de su apuesta resistente. Si bien otros aspectos de la obra de Lefebvre quedarán más relevados cuando aborde el concepto de estética del deterioro, me permito señalar que un elemento clave que el teórico francés le aporta a nuestra crítica es la concepción del lenguaje como expresión de una comunidad fundada en la praxis: "Estar juntos es hacer algo juntos [...] La palabra 'hacer' no debe entenderse en una acepción estrecha: la operación, la técnica. Implica una doble relación activa: entre los miembros del grupo (la praxis propiamente dicha) y con la materia y el objeto (la poiesis)" (120).

¿Cuáles serían, entonces, las estrategias culturales que caracterizan a la cultura de la resistencia y a qué referentes teóricos reenvían? En lo fundamental, se moverían en el siguiente terreno:

$[\mathrm{L}]$ os artistas que corresponden a la cultura de la resistencia $[\ldots]$ rechazaron la modernización refleja como una forma de impostura, pero se sirvieron de los materiales lingüísticos modernos que se conocieron a través de ella. Sortearon asimismo la degradación cultural, pero exploraron a conciencia esa zona, considerándola una rica cantera de elementos aprovechables. Las mejores obras de las artes plásticas continentales funcionaron en este orden subversivo espontáneo, no programado por ningún grupo de poder (Traba, "La cultura" 46).

Me interesa relevar aquí cómo Traba pasa, en unas cuantas líneas, por los aportes de la antropología de la civilización de Darcy Ribeiro (El proceso civilizatorio; Las Américas) para luego remitir a la semiótica y, luego, a la retórica de la crítica cultural, que ve al arte como subversión de lo establecido. Así, es capaz de sacarle el lustre políticocultural a un conjunto de creaciones dispersas, como las de Matta, Lam, Tamayo, Obregón, Szyszlo o Botero, entendiéndolas como la respuesta a una tendencia que, entre los dominados, asimila la conciencia del dominador, como el ejercicio en que se devuelve el boomerang hacia las metrópolis mediante un mirar nuevamente aquello que conforma el acervo de "lo propio", de lo vivido efectivamente.

A propósito de este problema -el de la asimilación de la conciencia externa-, cabría apuntar el desarrollo de ciertos conceptos clave trabajados por Ribeiro para entender la categoría de cultura de la resistencia. Tal vez el más importante de ellos sea el par cultura auténtica/cultura espuria. El primero hace referencia a las "culturas más integradas internamente y más autónomas en el comando de su desarrollo", mientras que el segundo concepto se predica de aquellas "culturas traumatizadas y correspondientes a sociedades sometidas a vínculos externos de dominación que se vuelven dependientes de decisiones ajenas y cuyos miembros están más sujetos a la alienación cultural, o sea, la internalización de la visión del dominador sobre el mundo y sobre sí mismos" (Las Américas 30). Entran en juego las nociones de autonomía y heteronomía, pensadas en virtud de procesos históricos de dominación colonial que 
tienen una dimensión constitutiva en el plano de la cultura. Ahora bien, el hecho de reconocer que América Latina se encuentra más bien en el polo espurio no implica, de suyo, establecer que dicha situación sea homogénea (Ribeiro, Las Américas 31). Se destaca aquí "la presencia de elementos de tecnología más alta cuya ausencia en [la] propia cultura la hiciera caer en vasallaje. Estos contenidos progresistas no se configuran, empero, como una infraestructura tecnológica de una economía autónoma, sino como implantaciones auxiliares del centro rector, que de él depende para su renovación y mejoría" (Ribeiro, Las Américas 32) ${ }^{9}$.

Sin embargo, y como puede colegirse del segmento de "La cultura de la resistencia" comentado más arriba, el resistir no tiene una dimensión puramente negativa. Por el contrario, el proceso creativo que caracterizaría a los intentos por quebrar la dependencia del continente partiría de una transmutación de la experiencia real de un individuo inserto en su entorno, al punto en que se originan una multiplicidad de respuestas al desborde que lo real impone por sobre los límites de las formas adaptadas de los centros metropolitanos (Traba, "La cultura" 53-55). En sus aspectos afirmativos, entonces, la cultura de la resistencia se expresa como esta apertura al exceso insoslayable de la realidad que se manifiesta en y a través de la obra: "A veces el artista o el escritor de la cultura de la resistencia no persigue expresamente el lenguaje simbólico o metafórico, sino que actúa como transmisor de una realidad cuya riqueza, variedad y peculiaridad es demasiado atractiva para poder desprenderse de ella" ("La cultura" 54). A partir de esta sumisión productiva al mandato de la experiencia es que se arraiga una de las marcas más pregnantes de la resistencia cultural latinoamericana: la mitologización. En un gesto que parece tener un ojo en Lévi-Strauss y otro en la tradición amplia de la cultura letrada latinoamericana que ha tematizado el mito (desde Mariátegui hasta Arguedas, pasando por Rulfo, Paz y García Márquez), Traba pone sus fichas en las obras que, para afirmar una particularidad continental, se hacen de los procedimientos mitológicos: tiempos recurrentes, espacios cerrados, indistinción entre lo "mágico" y lo "mundano":

En este prestigio de la mitología se comprueban dos hechos: primero, la voluntad de trascender el medio circundante, el nativismo chato y contingente, y hasta la presencia opaca y encenizada del indio sobreviviente; el segundo hecho es la capacidad de arrancar la realidad nacional del subdesarrollo, y trasponerla a un nivel mágico, o mítico, o puramente imaginativo, que se considera muy por encima de la posible imitación de tareas propuesta por la sociedad altamente industrializada (Dos décadas 99).

9 Puede anotarse, a propósito del problema de la tecnología como signo de dominación, una afinidad entre Ribeiro y algunas ideas de Marcuse (aun cuando en la obra de este exista una consideración filosófica particular sobre la tecnología que la circunscribe al vocabulario de la Teoría Crítica; el problema, en parte, pasa por la relación entre sujeto y dominio de la naturaleza, y tiene connotaciones que no se encuentran del todo en el pensamiento de Ribeiro, que evalúa la tecnología como parte de su teoría antropológica evolutiva). En efecto, es la misma Traba quien propone esta afinidad, aunque no de forma explícita, en su noción de tecnología ideológica (Cf. Dos décadas 58-60). 
Aquí salta a la vista que no hay una vía única prescrita por Traba, ni tampoco que el mito sea una característica esencial de toda plástica latinoamericana; más bien, lo que se releva es la ineluctable contaminación de la obra por la experiencia, no obstante -o, más bien, a causa de- haber sido transfigurada, descompuesta y recompuesta de algún modo que le confiere su status de obra de cultura ${ }^{10}$.

Uno de los objetos artísticos que más productivamente funcionan junto a la categoría de cultura de la resistencia es la producción plástica de una generación de pintores y escultores que funcionan dentro de lo que denomina "áreas cerradas", espacios de más débil vinculación cultural y socio-económica con los centros metropolitanos ${ }^{11}$. Quisiera cerrar este apartado recurriendo a un ejemplo del despliegue conceptual trabiano en la pintura de Alejandro Obregón. En su obra se realizaría una construcción cultural que, mediante la apropiación de los recursos plásticos de la pintura moderna tal y como se desarrollaron en Europa y Estados Unidos, logra establecer una imagen con dimensiones comunicativas, precisamente por ser el resultado de una elaboración de la experiencia ${ }^{12}$ (mítica). Traba define así a este proyecto como

[L]a trasposición de la geografía colombiana a la pintura (...) geografía y no paisaje, porque se trata de una cosa distinta, de una elaboración, rudimentaria, intelectual y sentimental, para dar con las formas paradigmáticas de un país cuya extraordinaria suerte natural hace aún más agraviante su problemática social y su flotante subhumanidad (Dos décadas 94).

El interés de la crítica argentina-colombiana es, por ende, una modulación que parte de lo regional como el horizonte que coordina una creación moderna consciente de su posición dentro de un entramado dependiente, y que resiente la modernización voraz que arrasa con la racionalidad específicamente plástica de un arte que aspira, en su mitología, a encarnar una comunidad subordinada que se encuentra en proceso de liberación. Aunque indirecta, esta encarnación, para Traba, no puede sino reclamar el carácter residual de lo pictórico como un recurso de resistencia al proceso artístico hegemónico que inunda a América Latina en los sesenta; el arcaísmo aparente de la construcción mítica como utopía contrahegemónica, testimonio de una modernización inexistente o, a lo sumo, inconmensurable con la industrialización metropolitana. En definitiva, el arte de la resistencia es, a este efecto, doblemente

10 Esta defensa del mito es una de las razones por las cuales se le achaca a Traba una postura esencialista en lo referido al arte latinoamericano (cf. Jara; Ramírez). Precisamente y en la medida que ella vuelve sobre la mediación ejercida por el artista es que tal lectura me parece errónea. Si algo caracteriza al esencialismo cultural es su afirmación de que determinadas obras, significados o prácticas son inevitables. Afirmación que se encuentra lejos de la crítica trabiana, como creo que las citas dejan en claro.

11 Para una discusión un poco más detallada de las categorías áreas cerradas y áreas abiertas ver Marambio (77-89).

12 Alinea, de este modo, la plástica de Obregón con la idea de forma como configuración, presente en Adorno: "Ley de la transfiguración de lo existente, la forma representa frente a esto la libertad [...]; no es Creación, pero sí el comportamiento objetivado de los seres humanos que imita a la Creación; por supuesto, no es una creación desde la nada, sino desde lo creado" (Teoría estética 194). 
resistente, pues batalla contra la dependencia estética y la económica reconvirtiendo la barbarie desde sus propios efectos, devolviendo la imagen propia entre los dominados y contra los dominantes.

\section{III}

La noción de estética del deterioro es, ante todo, un concepto cuya aplicación Traba no restringe al espacio latinoamericano. Por el contrario, forma parte de un instrumental que permite entender la globalidad del proceso de las artes plásticas contemporáneas, en particular las tendencias desarrolladas en Estados Unidos desde fines de los cincuenta: el pop, el minimalismo, así como las obras que se movían hacia a la desmaterialización ${ }^{13}$. El alcance espacial de la categoría le permite a Traba distinguir entre la historia acontecida en el corazón del mundo metropolitano y su contraparte latinoamericana, lo que parece producir una suerte de desdoblamiento de la categoría, capaz de dar cuenta -en forma diferenciada- de la actualización de un mismo proceso en polos distintos del globo. Por intermedio de esta amplitud conceptual es que se logra mantener la generalidad que enmarca las relaciones entre dos sociedades; la estética del deterioro es efectiva en Latinoamérica y en Estados Unidos, pero no de la misma manera, pues el despliegue de procedimientos y recursos artísticos en uno y otro contexto no logra tener el mismo significado. Con tal gesto es que Traba afirma la particularidad de la cultura latinoamericana: a pesar de que las relaciones imperialistas hagan operar a las artes plásticas de modo similar, subsiste el hecho de que hay un polo colonizador y otro colonizado, y que el sentido de una obra no puede desvincularse de estos marcos de referencia que constituyen el repertorio de recursos a los que un artista echa mano en el momento de la creación.

Sugiero que en este punto se producen anudamientos con al menos dos tradiciones europeas de pensamiento sobre el arte. De un lado, como ya apunté más arriba, la estética de Adorno, para quien "las fuerzas productivas en las obras de arte no son diferentes en sí de las fuerzas sociales, sino sólo al ausentarse constitutivamente de la sociedad" (Teoría estética 312) ${ }^{14}$. Al mismo tiempo, el territorio estético de Traba aparece signado por la sociología del arte de Pierre Francastel, en la cual la obra comparece bajo los auspicios -a veces más y a veces menos ex-

13 La reciente historiografía artística latinoamericana ha revisitado teóricamente al conceptualismo y la desmaterialización en la medida en que ha buscado establecer genealogías alternativas para el arte latinoamericano, que no lo subsuman a la línea de tiempo metropolitana. A este respecto, la obra y reflexión de Óscar Masotta han sido puntos cruciales para pensar desarrollos alternativos desde Latinoamérica. A modo de ejemplo, ver Longoni y Mestman ("Después del pop") y Luis Camnitzer.

14 Como debiera resultar evidente en lo que sigue de este ensayo, la problematización del vínculo arte/sociedad en la obra de Adorno es sumamente compleja. Sólo por remitirme a la Teoría estética, pueden revisarse los apartados "Lógica, causalidad, tiempo", "Finalidad sin fin", "Carácter doble del arte: fait social y autonomía" y "El carácter fetichista”, entre otros. Para discusiones más detalladas ver los trabajos de Shierry Weber Nicholsen y de Gillian Rose (especialmente 141-177). 
plícitos- de cierto estructuralismo: “[...] No cabe duda de que el signo figurativo es el punto de realización de una conducta no sólo práctica y manual sino también intelectual para cierta categoría de individuos a quienes se llama artistas [...] cuya misión es [...] producir e interpretar obras de un tipo determinado" (Sociología 11-12). Asimismo, sostiene Francastel que "un objeto artístico nunca es solamente estético. Se halla ligado a la realidad humana y social por un complejo conjunto de nexos" ("Arte, forma" 73). El pensamiento estético de la argentina-colombiana se fundamenta, en definitiva, en un puente problemáticamente tendido entre sociedad y arte, entre mundo y obra, que no recusa las tensiones que se manifiestan en cualquiera de los términos de la relación, sino que, por el contrario, las reconoce y explora en todas sus consecuencias. De este modo, queda habilitada para realizar una crítica de arte que salga de la pura descripción formal, a la vez que evita el reduccionismo sociológico, como ya he señalado. Tanto el concepto de cultura de la resistencia como el de estética del deterioro son parte de este esfuerzo por construir una tercera vía para la escritura sobre arte, postura a todas luces difícil de sostener en medio de un campo intelectual tensionado por un esteticismo incapaz de ponderar sus alianzas políticas y por una franja anti-intelectualista que subsumía a la revolución en la vanguardia político-militar (ver Giunta; Terán; Gilman). Participa, entonces, la categoría de estética del deterioro de un complejo mucho más amplio, que he delineado ya parcialmente. Para dar cuenta de dicho concepto propongo un breve itinerario, con tres hitos: en primer lugar, discutiré la caracterización de las sociedades metropolitanas -o "centros emisores", como los llama Traba- y la crisis del arte que en ellas se produce; en una segunda instancia daré cuenta de la contraposición entre lo que Traba denomina estética tradicional y la estética del deterioro; para finalizar, ofreceré algunos ejemplos del despliegue de la estética de deterioro en América Latina.

En la introducción de su polémico estudio Understanding Media, Marshall McLuhan sostiene que, merced a la electricidad, el tiempo y el espacio se han comprimido de tal manera que los valores y principios de la sociedad basada en la escritura estarían caducos: "Ya no es posible adoptar el rol desapegado y disociado [aloof and dissociated role] del letrado occidental" (4). Como consecuencia, se produce un colapso del mundo que lo reduciría a la famosa "aldea global". "Es este factor implosivo el que altera la posición del negro, el adolescente y algunos otros grupos. Ya no pueden ser contenidos, en el sentido político de la asociación limitada. Ahora están involucrados en nuestras vidas, como nosotros en las suyas, gracias a los medios eléctricos" (5). Con esta afirmación, McLuhan pone sobre la mesa los efectos reorganizadores que distintas tecnologías tienen sobre el conjunto de la sociedad. En particular, la comunicación a través de medios electrónicos permitiría prescindir de las formas políticas hasta entonces dominantes, lo que viene a señalar una transformación cualitativa de primer orden en los centros industrializados de mediados del siglo XX. Similares conclusiones, pero sin el evidente entusiasmo del 
teórico canadiense ${ }^{15}$, saca Herbert Marcuse de su análisis de las llamadas sociedades afluentes: "La independencia del pensamiento, la autonomía y el derecho a la oposición política están siendo privados de su función crítica básica en una sociedad que parece cada vez más capaz de satisfacer las necesidades de los individuos por medio de la forma en que se organiza" (1).

Tecnología y sociedad son presentadas como elementos cuyo vínculo aparece con igual nitidez para teóricos de signo distinto -es, a fin de cuentas, la carga ideológica y su crítica lo que diferencia a McLuhan de Marcuse-. La brutalidad con que se afirma esta complicidad técnica queda en evidencia al momento en que el primero discute los posibles efectos del medio televisivo en la asimilación de sociedades subdesarrolladas a patrones de conducta afines al capitalismo industrial de Occidente: "Culturas enteras podrían ser ahora programadas para mantener su clima emocional estable, de la misma forma en que hemos aprendido algo acerca de la mantención del equilibrio en las economías comerciales del mundo" $(28)^{16}$. Da la impresión de que el alcance omnicomprensivo de la tecnología en la sociedad contemporánea ha desarraigado un conjunto de relaciones sociales y valores previamente dominantes, siendo el arte y la cultura afectadas, también, en este proceso.

La característica novedosa de hoy es el achatamiento del antagonismo entre cultura y realidad social por medio de la obliteración de los elementos oposicionales, ajenos y trascendentales de la alta cultura en virtud de los cuales constituía otra dimensión de la realidad. Esta liquidación de la cultura bidimensional ocurre, no a través de la negación y rechazo de los "valores culturales", sino a través de su completa incorporación al orden establecido, a través de su reproducción y exhibición a escala masiva (Marcuse 57).

Marcuse actualiza la crítica desarrollada desde hace décadas ya a la industria cultural por los miembros de la Escuela de Frankfurt ${ }^{17}$. Tras publicarse la Dialéctica de la Ilustración en 1947, el concepto de industria cultural ha tenido un largo recorrido, originando interpretaciones de índole diversa, circulando de forma más amplia en América Latina producto de la publicación aislada del capítulo dedicado a la indus-

15 El contrapunto -a la vez que cierta cercanía en lo referido a los diagnósticos- entre las posturas de McLuhan y Marcuse ha sido ya notado por Marshall Berman. Si el primero es presa del optimismo tecnológico que inauguran los futuristas y que luego continúan las ciencias sociales funcionalistas, el segundo es teóricamente deudor de la noción de "jaula de hierro" acuñada por Max Weber. Ver Marshall Berman (13-17).

16 Este descaro resulta mucho más escandaloso -y por ende, más inexcusable, si es posible- cuando lo comparamos con la advertencia de Marcuse a propósito de la modernización programada del Tercer Mundo: "(...) parece que el desarrollo sobreimpuesto de estos países traerá un período de administración total más violenta y más rígida que aquella por la que han pasado las sociedades avanzadas, las que pueden sostenerse sobre los logros de la era liberal. En resumen: las áreas atrasadas de seguro sucumbirán a una de las varias formas de neocolonialismo o un sistema más o menos terrorista de acumulación primitiva" (47).

17 "La industria cultural integra intencionalmente a sus consumidores desde arriba. En detrimento de ambas, junta a la fuerza a las esferas del arte alto y bajo, separadas por miles de años. La seriedad del arte alto es destruida en la especulación sobre su eficacia; la seriedad del bajo perece con las limitaciones civilizatorias impuestas a la resistencia rebelde que le era inherente mientras el control social no fuese aún total" (Adorno, "Culture Industry" 12). 
tria de la cultura (cf. Varela 766) ${ }^{18}$. Por su parte, Traba no recurre a una sola fuente de todo el equipo frankfurtiano, sino que amalgama y traduce de manera libre las consideraciones que versan sobre el cambio en los centros metropolitanos, que han visto el predominio del arte mercantilizado por sobre una estética perdurable, lo que viene a reforzar la dominación inherente a tal sistema en virtud de la alianza entre subordinación y técnica: "La tecnología, hábilmente convertida en ideología por quienes la necesitan manejar como instrumento de poder, ha penetrado la unidad cultural de la sociedad de consumo y la ha atomizado" (Dos décadas 59).

Un arte producido en estas circunstancias vendría a verificar, en la mayor parte de los casos, un mero saludo a la bandera que permita mantener las cosas como están. Tal es la fuerza de la "sociedad administrada" que describe Marcuse; fuerza que parece refrendar el mismo McLuhan al sostener la función farmacológica del arte experimental que, por vía de su imbricación con el ritmo de cambio tecnológico, nos apresta para la transformación de los medios: "De cualquier modo, en el arte experimental a los hombres se les dan las especificaciones exactas de la violencia venidera para sus propias psiquis por parte de sus contra-irritantes o tecnología" (66). Proceso similar ocurre con la publicidad, la cual "es un intento crudo de extender los principios de automatización a cada aspecto de la sociedad. Idealmente, la publicidad apunta al objetivo de una armonía programada entre todos los impulsos y aspiraciones y empresas humanas" (McLuhan 227). Por todos lados parece extenderse la degradación de valores culturales característicos de la tradición moderna -en cualquiera de sus formas-, al punto en que no hay distinción entre alta y baja cultura, y sólo quedan disponibles los productos de la sociedad de consumo bajo la forma del kitsch. Señala Clement Greenberg: "El kitsch, en virtud de una técnica racionalizada que recurre a la ciencia y la industria, ha borrado esta distinción en la práctica" (13). Da la impresión de que, en su desbocada masificación del consumo, la sociedad industrial disolvió las fronteras entre dominios que se mantenían a perfecta y calculada distancia ${ }^{19}$. Sobre los productos kitsch del mundo industrializado, Traba se pronuncia remitiendo a Eco y Adorno:

[S]on [objetos] conservadores, es decir se resisten tenazmente a la creación y al cambio; responden a la ley de la oferta y la demanda, y por eso se convierten de buena gana en productos condensados y nivelados; hacen digerir cosas fáciles, igualan por el nivel de entretenimiento, cumplen una función satisfactoria, complacida y acrítica, buscan símbolos y mitos de rápida universalidad, sirven de instrumento al paternalismo, trabajan sobre los lugares comunes ("La pintura" 297).

18 Una síntesis sobre las principales características de la reflexión sobre estética y cultura en los miembros de la Escuela de Frankfurt puede encontrarse en David Held (75-109).

19 "Siempre ha existido, por un lado, la minoría de los poderosos -y por ende, los cultos- y, por otro, la gran masa de los explotados y pobres -y por ende, los ignorantes-. La cultura formal siempre ha pertenecido a los primeros, mientras que los últimos han tenido que contentarse con la cultura folk o rudimentaria, o el kitsch" (Greenberg 16). 
Ahora bien, Traba realiza una lectura sutil de las tendencias ya mencionadas, cuidándose de limitar su efectividad a los espacios metropolitanos. De este modo, la penetración de la industria cultural en el ámbito estético, la "desublimación represiva" que anula el potencial utópico-crítico del arte (Cf. Marcuse, 61), la indistinción entre alta y baja cultura, la presencia, al parecer, ubicua de los medios de comunicación en la vida cotidiana y la hegemonía del kitsch, vienen a ser todas facetas de una crisis que atañe específicamente a las sociedades industrializadas y no a los países subdesarrollados que constituyen la región latinoamericana (Traba, "La cultura" 38-39). Visto en su globalidad, es este proceso el que da origen a la estética del deterioro, y su caracterización permitirá -como mostraré más adelante- argumentar que la hegemonía de dicha estética en América Latina no es sólo “inauténtica”, sino además una señal inequívoca de colonialismo cultural.

En efecto, el término estética de deterioro viene a designar la manifestación en el arte de un proceso mayor de transformación histórico-social, y Traba lo señala con claridad:

El resultado más ostensible de la "tecnología ideológica" sobre las artes plásticas norteamericanas es el reemplazo de lo que podríamos llamar estética tradicional por la estética del deterioro. Si el valor culminante de la estética tradicional consiste en lograr la permanencia de la obra de arte superando las contingencias de la época y de la moda para aplicarse a coordenadas de estilo, la estética del deterioro, por el contrario, descarta o desafía abiertamente ambas concepciones. La cuestión consiste en no durar y en no establecer pauta alguna (Dos décadas 60).

Abjura, en consecuencia, esta estética de cualquier posibilidad de pervivencia o comunicabilidad, con lo que responde a la disolución de lo que Traba, siguiendo a Lefebvre, denomina "código general". "La ausencia de un código general (compensada por la introducción de códigos parciales, que en rigor organizan imperativamente una red, como por ejemplo el código carretero) permite las trampas, los trucos de códigos y de mensajes, la introducción de elementos equívocos, mal conocidos o desconocidos" (Lefebvre 114). Con un código general en retirada -o por completo ausente-, campea la fragmentación social que caracterizaría al mundo metropolitano, al punto en que se mina la capacidad de producir sentido por haberse reemplazado el signo por la señal; la ausencia de sentido se manifiesta como apariencia de sentido (Ver Lefebvre 118-121). De este modo, la estética del deterioro se nutre de una crisis más general que afecta las capacidades expresivas de una sociedad determinada; empuja al arte hacia una zona de asimilación por negarle la posibilidad de restarse del intercambio mercantil y de sus pautas de obsolescencia: "Sin posibilidad de permanecer [...], el arte se autocondena al mismo destino que los demás productos de la sociedad de consumo; apenas se gasta, cubre una espectativa $[s i c]$ y satisface episódicamente a su cliente, desaparece" (Traba, Dos décadas 61). 
Bajo los marcos de la estética del deterioro, el arte se asimila a las lógicas del proceso productivo en el capitalismo avanzado y, con ello, le quita el eje de su poder: la autonomía que se reclama en el seno mismo de la sociedad moderna. De acuerdo a la argentina-colombiana, el resultado no es sino "un elemento catártico perfectamente prefabricado cuyo triple permiso -el de divertir, liberar, destruir- se ha ejercido [...] con terrible y pasmosa regularidad" (Dos décadas 61). Me parece que en estos gestos es posible advertir la consonancia con partes notorias de la estética de Adorno, para quien "las obras del cambio absoluto, de la pluralidad sin relación con la unidad, se vuelven [...] indiferentes, monótonas, uniformes" (Teoría estética 254). Al mismo tiempo, critica Adorno la tendencia al experimentalismo que campea en las vanguardias entregadas a la participación y lo lúdico: "Hoy, la cultura oficial le concede zonas especiales a lo que (desconfiada y con la esperanza de que fracase) declara un experimento, con lo cual lo neutraliza" (Teoría estética 57) ${ }^{20}$. Por su parte, Traba sostiene que "[el] juego es un aliado de los sistemas enajenantes, puesto que les facilita los antídotos o los medios de desintoxicación. No es una coincidencia que el entusiasmo suscitado por los juegos-catarsis y su adopción por el sistema haya sobrevenido al tiempo con las grandes crisis" (Dos décadas 100).

Sin embargo, creo que es necesario prevenirse contra una inscripción automática de Traba en algo así como un paradigma estético adorniano, al menos por tres motivos. La primera razón, evidente para quienes estén familiarizados con la obra de Adorno, se refiere al despliegue propiamente dialéctico y contradictorio de su pensamiento: no hay tal cosa como la estética que se corresponda con su obra, ni tampoco un programa protocolizado al cual pudiera alguien subscribir ${ }^{21}$. En otro nivel, la reflexión adorniana contrapone a la obra como nexo de sentido a la disolución de ese nexo (nuevamente recompuesto por el principio de montaje y construcción) por la misma obra en el modernismo. Se dibuja así un recorrido que insiste en la negatividad, al punto en que la obra debe enfrentarse a su propio perecer; aferrarse a la duración vendría a significar nada más que el clasicismo, puesto que el despliegue procesual de la obra se identifica con su desaparición ${ }^{22}$. En la medida que Traba busca esbozar un programa "afirmativo" -no obstante utilizar la noción negativa de resistencia-, su asimilación al pensamiento no-identitario que construye Adorno resulta, al menos, problemática. A este respecto, llama la atención el hecho de que este último distinga entre la mera negación de sentido y su presencia en ciertas obras calificadas de absurdas, lo que constituye una "negación determinada" en la línea que él reivindica ${ }^{23}$. Por

20 También señala: "La irresponsabilidad absoluta [de las obras] las reduce a fun" (Teoría estética 59).

21 Sostiene Jay: "Adorno hubiera realizado una objeción de principio a cualquier intento de volver indoloramente accesible su pensamiento a una audiencia amplia. La verdadera filosofía, le gustaba insistir, es el tipo de pensamiento que resiste la paráfrasis" (Adorno 11).

22 Véase las secciones "La experiencia estética es procesual" y "Carácter perecedero" en Teoría estética.

23 Cf. Adorno (Teoría estética 211). También: "El umbral entre el arte auténtico que carga con la crisis del sentido y un arte resignado que está formado por enunciados protocolares (literal y figuradamente) es que en las obras 
último, vale la pena considerar un punto de orden cronológico: la Teoría estética se publica hacia principios de los setenta, y su incorporación a Dos décadas vulnerables resulta difícil de probar. Si bien la obra de Traba da cuenta de lecturas y traducciones heterodoxas ${ }^{24}$ a la vez que sorprendentemente rápidas -teniendo en cuenta su notoria movilidad tras la salida de Colombia en los sesenta-, me parece poco probable que haya estado en condiciones de hacer una integración detallada de la obra última de Adorno a su trabajo de los setenta.

En síntesis, podría señalarse que la estética del deterioro se opone a la estética tradicional (término que no deja de ser ambiguo, pues no queda claro si es que Traba se refiere a una estética idealista o si está pensando, antes, en los presupuestos del arte moderno en general ${ }^{25}$ ) por cuanto se entrega al abandono del sentido y el empobrecimiento del arte como lenguaje, al tiempo que desarrolla una temporalidad que sólo tiende a la autodestrucción y al descarte en los ritmos de la moda. Cuando el arte pop fragmenta la visión, en su imitación de la gráfica del cómic, acontece para Traba la negación sin más de la lógica constructiva modernista y se nos deja en un estado de desarme progresivo, sin herramienta alguna para construir un sentido comunitario a partir de las imágenes:

[...] hasta qué punto el fragmento de lenguaje desmembrado de su estructura de sentido, ocupando plásticamente un espacio estrecho [puede] ser interpretado con un público dotado de espíritu crítico capaz de experimentar la sensación de empobrecimiento y la superficialidad de tal imagen; y hasta qué punto tal imagen no [crea] en el espectador un progresivo estrechamiento de su propia habla y lo [empuja] a adoptar como lenguaje corriente esa suma de fragmentos incoherentes [...] [E]levando los fragmentos a categoría de obra de arte, [los manipuladores de cultura] han logrado separar al artista de toda preocupación conceptual y significante; lo ha convertido en un producto, cuyo suministro atiende celosamente, así como atiende que la demanda se produzca en el momento preciso y también en el momento preciso se cancele, cuando el mercado está saturado de determinado fragmento (Dos décadas 146-147).

Si antes fue lenguaje, la pintura quedaría ahora arrojada a la mera objetualidad, a la visualidad pura o al simple descarte de lo caduco. No es otra cosa que la pasión por los neologismos y la programación de las tendencias de acuerdo al furor de estar in (Traba, "Replanteo" 224). Una máquina devoradora pasa por encima de los intentos

significativas la negación del sentido se configura como algo negativo, mientras que en las otras obras se copia de una manera torpe, positiva" (Adorno, Teoría estética 207).

24 Sólo dos ejemplos, ambos de principios de los setenta: la referencia a Benjamin en "La pintura como medio de comunicación" y una cita de Eros y civilización que aparece hacia el final de Dos décadas vulnerables y que difiere lo suficiente de la versión de Juan García Ponce, primera edición castellana, como para sostener que sea Traba quien traduce. Ver Traba ("La pintura" 295; Dos décadas 219-220).

25 Cf. Traba ("La rebelión" 191). El texto es un fragmento de La rebelión de los santos. 
por fijar un lenguaje y, más allá de la angustia existencial que ello pudiera provocar, el efecto más escandaloso es que la crisis del arte que experimentan Estados Unidos y Europa se replica sin más en Latinoamérica.

La expresión continental de la estética del deterioro tiene múltiples vías. Una de las más evidentes es lo que Traba denomina way out, o "la tendencia a formas experimentales dentro del programa del arte actual impartido por los artistas de Estados Unidos" ("Puerto Rico" 177). Programa que se patentiza en Puerto Rico como "el camino de la redención del pecado de ser isleños, mediante la escapada hacia afuera, el asimilismo incondicional de las corrientes norteamericanas" (Dos décadas 173). Salta a la vista el carácter colonial de dicha operación, máxime si es que, como Traba, nos ubicamos en la sintonía del equipo intelectual que ha tematizado y discutido la relación entre las culturas metropolitanas y la creación latinoamericana. En concreto, la crítica sentencia: "si los artistas del continente aceptan sin reservas las propuestas formuladas por los otros, terminarán por engrosar múltiples líneas de satelismo [...] si la rendición a las propuestas es incondicional [...] refrendan su condición de colonizados culturales" ("Puerto Rico" 177).

Otra marca de la presencia de la estética del deterioro en América Latina es la sucesión vertiginosa de modas y estilos, incluso para un mismo artista, en el transcurso de unos pocos años. Al comentar las bienales y salones de los sesenta, Traba afirma: "Distribuidos oscuramente por el espacio estrecho de cada moda; lanzados bien al óptico como al 'pop-art', como al cinetismo o los objetos, son artistas sin contexto" (Dos décadas 167). El ejemplo más sobresaliente de esta tendencia es, sin duda, la escena argentina experimental, la que manifiesta "la servidumbre a la moda", "la búsqueda de lo escandaloso e impactante", "la sumisión a las reducidas elites vanguardistas" y la subordinación "a la juventud y el pavor de envejecer" (Dos décadas 191). Sin posibilidad de construir una propuesta de estilo o un modelo de visión transmisible en obra, las vanguardias quedan sumidas en su propia temporalidad que tiende a la disolución de lo permanente -ellas mismas incluidas-. Con ello se cae, o bien en la frivolidad superficial (Traba, "La lucha" 263), o bien en la reducción de la obra a un objeto suntuario, una mera cosa sin ambiciones significantes (Traba, "Venezuela" 272, 276). Se pierden, de este modo, tanto las dimensiones plásticas como las comunicables en la obra de arte, y en ello no acontece otra cosa que el hacer como si el colapso de arte, ocurrido por razones que atañen a la particularidad histórica de las sociedades industrializadas, hubiese ocurrido también -y del mismo modo- en Latinoamérica, en circunstancias que las dichas razones no se encuentran ni cerca de constatarse en el continente.

A lo largo de este ensayo he intentado reconstruir las herramientas conceptuales que se despliegan en la obra de Marta Traba. He buscado, en el camino, apuntar cuáles son los instrumentos principales, cuáles las categorías de mayor relevancia y en qué momentos específicos los conceptos se transmutan para llevar a cabo una reinterpretación o una relectura que permita la emergencia de un pensamiento original, capaz 
él mismo de lanzarse sobre los objetos de los que aspira a dar cuenta. Mi enfoque en las nociones de cultura de la resistencia y estética del deterioro se debe al peso que tienen dentro de la obra de Traba y a que, a su modo, son los ejemplos de categorías propias de su pensamiento en las cuales más fructíferamente se reformulan conceptos tomados "de otro lado". La amalgama es, valgan verdades, heterogénea, como lo son también los objetos discutidos a través de ella. Quizás ahí sea donde resida la mayor fuerza de este pensamiento: en la audacia teórica, en los gestos desenfadados que leen con voracidad el presente con miras a salvarlo de su despeñamiento en el vacío del vanguardismo experimental.

\section{Referencias}

Adorno, Theodor W. Crítica cultural y sociedad. Trad. Manuel Sacristán. Barcelona: Ariel, 1969. Medio impreso.

---.“Culture Industry Reconsidered”. New German Critique 6 (Otoño 1975): 12-19. Medio impreso.

---. “Teoría estética”. Obra completa 7. Trad. Jorge Navarro Pérez. Madrid: Akal, 2004. Medio impreso.

Bal, Mieke. Conceptos viajeros en las humanidades. Una guía de viaje. Trad. Yaiza Hernández Velázquez. Murcia: CENDEAC, 2009. Medio impreso.

Bazzano-Nelson, Florencia. "Cambios de margen: las teorías estéticas de Marta Traba”. Dos décadas vulnerables en las artes plásticas latinoamericanas, 1950-1970 de Marta Traba. Buenos Aires: Siglo XXI, 2005. Medio impreso.

---. “Theory in Context: Marta Traba's Art-critical Writings and Colombia, 19451959”. Tesis de Doctorado en Historia del Arte, Universidad de New Mexico, Albuquerque, 2000.

Berman, Marshall. Todo lo sólido se desvanece en el aire. La experiencia de la modernidad. Trad. Andrea Morales Vidal. Madrid: Siglo XXI, 1988. Impreso.

Berríos González, Pablo. "Estrategias de inserción del arte latinoamericano: el internacionalismo en Jorge Romero Brest y el latinoamericanismo en Marta Traba”. Tesis de Magíster en Estudios Latinoamericanos, Universidad de Chile, 2011. Bourdieu, Pierre. The field of cultural production. Essays on art and literature. New York: Columbia University Press, 1993. Medio impreso.

Camnitzer, Luis. "La impropiedad histórica del conceptualismo en Latinoamérica". Versions and Inversions. Perspectives on Avant-Garde Art in Latin America. Héctor Olea y Mari Carmen Ramírez (Eds.). New Haven/London: Yale University Press/International Center for the Arts of the Americas, 2006. Medio impreso. Francastel, Pierre. “Arte, forma, estructura”. Estructuralismo y estética. José Sazbón (Ed.). Buenos Aires: Nueva Visión, 1971. Medio impreso.

---. Sociología del arte. Trad. Susana Soba Rojo. Madrid: Alianza, 1975. Medio impreso. 
Gilman, Claudia. Entre la pluma y el fusil: debates y dilemas del escritor revolucionario en América Latina. Buenos Aires: Siglo XXI, 2012. Medio impreso.

Giraldo, Efrén. Marta Traba: crítica del arte latinoamericano. Medellín: La Carreta, 2007. Medio impreso.

Giunta, Andrea. Vanguardia, internacionalismo y política. Arte argentino en los años sesenta. Buenos Aires: Siglo XXI, 2008. Medio impreso.

Greenberg, Clement. Art and Culture. Critical Essays. Boston: Beacon Press, 1965. Medio impreso.

Held, David. Introduction to critical theory: Horkheimer to Habermas. Berkeley/Los Angeles: University of California Press, 1980. Medio impreso.

Horkheimer, Max y Theodor W. Adorno. Dialéctica de la Ilustración: fragmentos filosóficos. Trad. Juan José Sánchez. Madrid: Trotta, 2006. Medio impreso.

Jara Parra, Natalia. "Los proyectos críticos de Marta Traba y Nelly Richard: Trayectos de la escritura sobre el arte en Latinoamérica”. Tesis de Magíster en Teoría e Historia del Arte, Universidad de Chile, 2012. Medio impreso..

Jay, Martin. Adorno. London: Fontana, 1984. Medio impreso.

---. "Fieldwork and Theorizing in Intellectual History: A Reply to Fritz Ringer". Theory and Society 19 (Junio 1990): 311-321. Medio impreso.

---. “Two Cheers for Paraphrase. The Confessions of a Synoptic Historian”. Stanford Literature Review 3 (Primavera 1986): 47-61. Medio impreso.

Koselleck, Reinhart. Vergangene Zukunft. ZurSemantik geschichtlicher Zeiten. Frankfurt: Suhrkamp, 1989. Medio impreso.

Lefebvre, Henri. Lenguaje y sociedad. Trad. Floreal Mazía. Buenos Aires: Editorial Proteo, 1967. Medio impreso.

Longoni, Ana y Mariano Mestman. Del Di Tella a “Tucumán Arde”. Vanguardia artística y política en el 68 argentino. Buenos Aires: EUDEBA, 2008. Medio impreso.

---. “Después del pop, nosotros desmaterializamos': Oscar Masotta, los happenings y el arte de los medios en los inicios del conceptualismo". Escritos de vanguardia. Arte argentino de los años '60. Inés Katzenstein (Ed.). New York/Buenos Aires: The Museum of Modern Art/Fundación Espigas/Fundación Proa, 2007. 160179. Medio impreso.

Marambio de la Fuente, Matías. "Campo intelectual y artes visuales: Marta Traba y la formación de una crítica de arte latinoamericano”. Tesis de Magíster en Estudios Latinoamericanos, Universidad de Chile. 2013. Medio impreso.

Marcuse, Herbert. One dimensional man. Studies in the Ideology of Advanced Industrial Society. London: Routledge \& Kegan Paul, 1964. Medio impreso.

McLuhan, Marshall. Understanding Media: The Extensions of Man. New York/London: McGraw-Hill, 1965. Medio impreso.

Pizarro, Ana (compiladora). Las grietas del proceso civilizatorio: Marta Traba en los sesenta. Santiago: CEXECI/LOM/IDEA/Wellesley College, 2002. Medio impreso. 
Rama, Ángel. La ciudad letrada. Santiago: Tajamar, 2004. Medio impreso.

---. Rubén Darío y el modernismo: circunstancia socioeconómica de un arte americano.

Caracas: Ediciones de la Biblioteca de la Universidad Central de Venezuela, 1970. Medio impreso.

Ramírez, Mari Carmen. "Sobre la pertinencia actual de una crítica comprometida". Dos décadas vulnerables en las artes plásticas latinoamericanas, 1950-1970 de Marta Traba. Buenos Aires: Siglo XXI, 2005. Medio impreso.

Ribeiro, Darcy. El proceso civilizatorio: etapas de la evolución sociocultural. Caracas: Ediciones de Biblioteca, Universidad Central de Venezuela, 1970. Medio impreso. ---. Las Américas y la civilización. Proceso de formación y causas del desarrollo desigual de los pueblos americanos. Caracas: Biblioteca Ayacucho, 1992. Medio impreso.

Ridenti, Marcelo. "Artistas e intelectuales brasileños en las décadas de 1960 y 1970: cultura y revolución". Historia de los intelectuales en América Latina, vol. II. Los avatares de la "ciudad letrada" en el siglo XX. Carlos Altamirano (Dir.). Buenos Aires: Katz, 2010. Medio impreso.

Roitman, Marcos. Pensar América Latina: el desarrollo de la sociología latinoamericana. Buenos Aires: CLACSO, 2008. Medio impreso.

Rose, Gillian. The Melancholy Science. An Introduction to the Thought of Theodor W. Adorno. London/New York: Verso, 2014. Medio impreso.

Schwarz, Roberto. “Cultura e política, 1964-1969”. Cultura e política. São Paulo: Paz e Terra, 2005. Medio impreso.

Serviddio, Fabiana. Arte y crítica en Latinoamérica durante los años setenta. Buenos Aires: Miño y Dávila, 2012. Medio impreso.

Terán, Óscar. Nuestros años sesentas. La formación de la nueva izquierda intelectual argentina, 1956-1966. Buenos Aires: Puntosur, 1991. Medio impreso.

Traba, Marta. "Arte en Puerto Rico: Crisis de valores; ¿La crisis como valor? Impugnaciones a la crisis". Mirar en América. Selección y prólogo Ana Pizarro. Caracas: Biblioteca Ayacucho, 2005. Medio impreso.

---. Arte latinoamericano actual. Caracas: Ediciones de la Biblioteca de la Universidad Central de Venezuela, 1972. Medio impreso.

---. “Artes plásticas latinoamericanas: la tradición de lo nacional”. Mirar en América. Selección y prólogo Ana Pizarro. Caracas: Biblioteca Ayacucho, 2005. Medio impreso.

---. “Crítica de Arte. El genio anti-servil”. Marta Traba. Emma Araújo de Vallejo (Comp.) Bogotá: Planeta/Museo de Arte Moderno de Bogotá, 1984. Medio impreso.

---. Dos décadas vulnerables en las artes plásticas latinoamericanas, 1950-1970. Buenos Aires: Siglo XXI, 2005. Medio impreso.

---. "La cultura de la resistencia". Mirar en América. Selección y prólogo Ana Pizarro. Caracas: Biblioteca Ayacucho, 2005. Medio impreso.

---. "La lucha contra el mimetismo en arte". Mirar en América. Selección y prólogo Ana Pizarro. Caracas: Biblioteca Ayacucho, 2005. Medio impreso. 
---. “La pintura como medio de comunicación”. Emma Araújo de Vallejo (Comp.). Bogotá: Planeta/Museo de Arte Moderno de Bogotá, 1984. 295-298. Medio impreso.

---. "La rebelión de los santos (Fragmento)". Mirar en América. Selección y prólogo Ana Pizarro. Caracas: Biblioteca Ayacucho, 2005. Medio impreso.

---. La rebelión de los santos. The rebellion of the santos. San Juan: Ediciones Puerto/ Museo de Santos, 1972. Medio impreso.

---. Los cuatro monstruos cardinales. México: Ediciones Era, 1965. Medio impreso.

---. "Replanteo desde cero". Mirar en América. Selección y prólogo Ana Pizarro. Caracas: Biblioteca Ayacucho, 2005. Medio impreso.

---. "Venezuela: cómo se forma una plástica hegemónica". Mirar en América. Selección y prólogo Ana Pizarro. Caracas: Biblioteca Ayacucho, 2005. Medio impreso.

Varela, Mirta. "Intelectuales y medios de comunicación". Historia de los intelectuales en América Latina, vol. II. Los avatares de la "ciudad letrada" en el siglo XX. Carlos Altamirano (Dir.). Buenos Aires: Katz, 2010. Medio impreso.

Weber Nicholsen, Shierry. Exact Imagination, Late Work. On Adorno's Aesthetics. Cambridge (MA)/London: MIT Press, 1997. Medio impreso.

Recibido: 07 octubre 2014

Aceptado: 27 febrero 2015 